\title{
Chapter 9 \\ Necessity of Adaptive Risk Management for Fisheries and Wildlife
}

\author{
Hiroyuki Matsuda
}

\begin{abstract}
The conventional theory of ecosystem and population management does not include the concept of risks. In risk management, survival rates and reproductive rates are taken into account in mathematical models describing conditions of the ecosystem, and uncertainty and environmental variation when measuring are taken into account when predicting population sizes. As a result, future prediction can only be made in a probabilistic way. Even when a general prediction is made about the future, therefore, it is rare to predict it accurately. Risk evaluation to show a prediction range with some allowance is required. Moreover, if management measures are predetermined, it is impossible to cope with a contingency. Adaptive management is therefore recommended as a management method to cope with uncertainties. This entails making a management plan based on unverified assumptions, continuously monitoring changes in the situation while implementing the plan, adjusting the management measures as required, and verifying the appropriateness of the assumptions used. It is important to predetermine how the measures will be adjusted and the assumptions verified. In this chapter, we highlight the difference in thinking before and after adaptive risk management is established, describing two cases: management of fisheries resources and wildlife management. Numerical calculations that appear in the figures in this chapter can be obtained through the website (http://risk.kan.ynu.ac.jp/matsuda/2014/SLER.html) and additional tests can be conducted with Excel files. Eager readers are strongly recommended to try this.
\end{abstract}

Keywords Deer management • Environmental impact assessment • Maximum sustainable yield $\bullet$ Uncertainty

H. Matsuda (訩

Graduate School of Environment and Information Sciences, Yokohama

National University, Yokohama, Japan

e-mail:matsuda@ynu.ac.jp 


\subsection{Is It Really Good for Fisheries to Reduce the Fish Population by Half?}

Capture fishery is an industry that enables humans to utilize wild seafoods as a resource, although, if overfished, breeding individuals become short of supply, making it impossible to secure the resources in the future. Accordingly, theories of resource management for appropriate fishing have long existed, although such theories typically ignore uncertainty. Of the conventional resource management theories, one such theory that ignores uncertainty is the classical theory of "maximum sustainable yield," or MSY, that appears in fisheries and wildlife management (Clark 1990).

In the classical MSY theory, we consider the reproduction curve as shown in Fig. 9.1. The formulae are shown at the end of the chapter. Biological populations are characterized by exponential growth in population numbers. Calculated in the simplest possible way, the per capita growth rate of a population would increase constantly, like the relationship between the amount of a bank deposit and interest (corresponding to the thin line in Fig. 9.1). However, the per capita growth rate in fact decreases as the stock increases because availability of food, habitat, and other resources becomes limited. Therefore, the surplus production, or the product of the population number and the per capita growth rate, forms a peak, corresponding to the bold curve in Fig. 9.1. In addition, the population does not increase beyond a threshold, which is called carrying capacity, denoted by $K$ in Fig. 9.1. The surplus production produces a curve of one crest in relation to the stock, as in Fig. 9.1, and with an intermediate stock level, the largest catches can be continued. The catches are called MSY, which in the case of Fig. 9.1 is 500,000 t. If the surplus production is larger than the catches (implied by the broken line in Fig. 9.1), the resources increase. If it is smaller, they decrease. The equilibrium stock biomass under the given catch level is the intersection of the surplus production curve and the catch

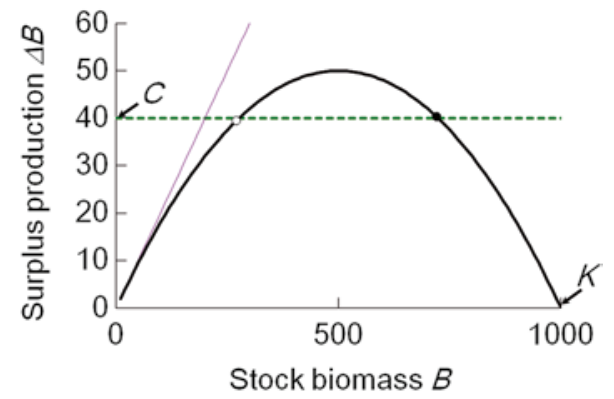

Fig. 9.1 The conceptual relationship between stock biomass $(B)$ and the surplus production (bold curve, $\Delta B$ ). $\Delta B$ is not proportional to $B$, unlike the thin line. $\Delta B$ reaches a peak at $B=500$ and $\Delta B$ becomes 0 at $B=1,000$, or carrying capacity $K$. If $\Delta B$ is larger than catch amount (broken line, $C$ ) the stock will increase. The stock will decrease if $\Delta B<C$. When $C<$ MSY or 50, there are stable and unstable equilibria that satisfy $\Delta B=C$, as shown by black and white circles, respectively 
amount, indicated by white and black circles in Fig. 9.1. If the yearly catches are smaller than the surplus production, the stock can be maintained at a high level. But if the resources approach the carrying capacity, the surplus production decreases, and this is not effective utilization. If, on the other hand, the catch amount is larger than the MSY, the stock decreases until extinction.

Catch at the MSY seems to be desirable from the viewpoint of sustainability, but fishery operators rarely know the true MSY level. Consequently, fishing is continued at a higher rate than the MSY, thus endangering many fish species.

Indiscriminate fishing damages not only the ecosystem but also economic profits over the long run. But why do attempts to increase production not succeed? The reasons are roughly divided into two types, as has long been pointed out in the science of fishery (Clark 1990); they include economic discounting and the tragedy of the commons. We do not explain these in depth here, however, as a bigger problem is the very assumption that the relationship as shown in Fig. 9.1 exists. The ecosystem is uncertain, non-equilibrium, and complex, yet in Fig. 9.1 these three factors are not considered. They will be explained sequentially below.

First, unless the reproduction relationship as shown in Fig. 9.1 is known, the MSY cannot be achieved. If $600,000 \mathrm{t}$ is continuously caught when the MSY is $500,000 \mathrm{t}$, the resources will be exhausted.

Next, Fig. 9.1 implies that if catches are continued at a certain level, the stock will settle at a certain amount, but this notion would in fact be laughed off by fishery operators. Populations of marine organisms fluctuate greatly depending on the environment, and while prohibition of fishing may even decrease populations in some cases, in other cases populations may not decrease even if many more individuals are caught. That is to say, the ecosystem is unsteady, and dynamic sustainability must be considered accordingly.This challenge can, however, be overcome by the adaptive management described later. In such cases, the prospects for resource recovery can be shown only in a probabilistic way. That is, risk management is required.

Finally, Fig. 9.1 relates only to the fish species to be caught, but an increased population of a certain fish species concerns not only the stock of that particular species, but also the population sizes of the organisms on which they prey and their natural enemies. The MSY theory does not consider this point, either. The effects of such interaction among species lead to a need to manage the ecosystem as a whole. Theoretically, this differs considerably from population management (Matsuda and Abrams 2004, 2006, 2013).

The southern bluefin tuna (Thunnus maccoyii) was intensively caught from the 1960 s to the 1980s, resulting in stringent fishery controls being agreed on by an international management organization, the Commission for Conservation of Southern Bluefin Tuna (CCSBT). They set a numerical target to recover the stock to its 1980 level by 2020, and in the latter half of the 1990s signs finally appeared to suggest that the resources would eventually be recovered. Then Japan substantially increased its catches without obtaining the consent of other countries, and was brought to the international court. Yet, after Japan reduced its catches again, southern bluefin tuna resources did not recover as expected. One reason was that the resource recovery prediction at the beginning was too optimistic (Mori et al 2001, see Fig. 9.2) 


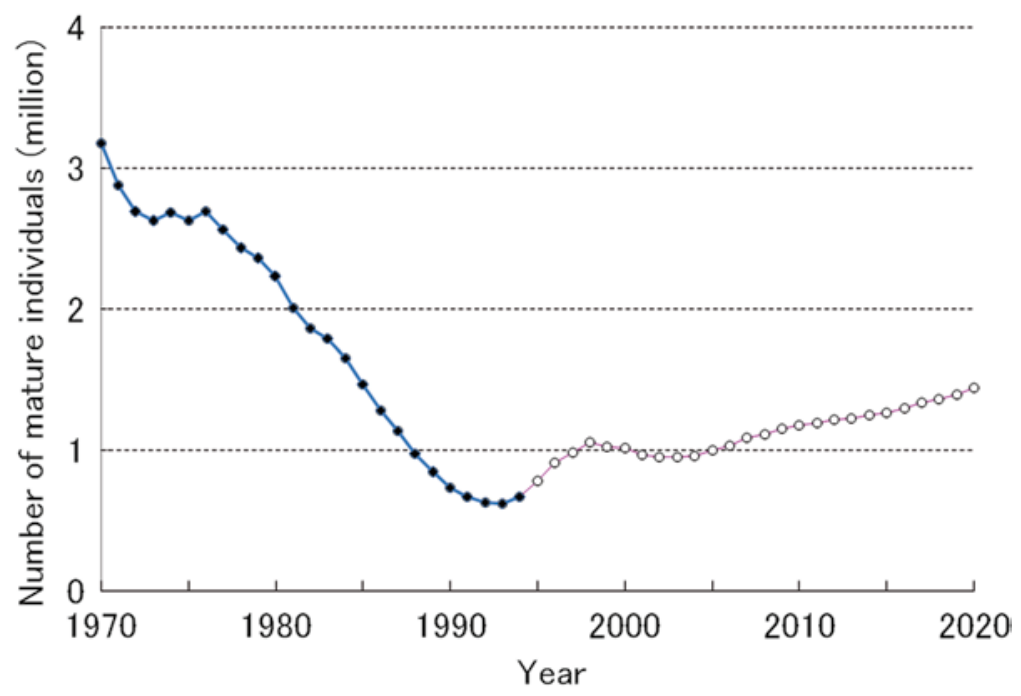

Fig. 9.2 Trends in the southern bluefin tuna population (Mori et al. 2001). Black circles and white circles are past estimates and medians of future projections, respectively

and the other reason was under-reporting of catches. Mori et al (2001) predicted that the stock would not monotonically increase even under the stringent fishery controls, which was indeed the case.

\subsection{Comparison Between Conventional Management Methods and Adaptive Management Considering Uncertainty}

To overcome the problems of uncertainty and non-equilibrium, the adaptive management method was proposed (Holling 1966; Walters 1986). This entails making a management plan based on unverified assumptions, continuously monitoring while implementing the plan, adjusting the management measures as the situation evolves, and verifying the assumptions used.

The idea of adaptive management has been introduced in the recent management of marine resources. An example is shown in Fig. 9.3. With the same mathematical model shown in Fig. 9.1, the prediction when catching is continued at the exploitation rate (0.05) to realize the theoretical MSY is as shown in Fig. 9.3a. If, however, the true MSY is not known, the exploitation rate often becomes excessive. Even if the figure shows that the stock changes along a smooth curve, therefore, it is not persuasive because it does not accord with the actual experience of fishery operators. They know that the stock fluctuates greatly year by year because of environmental changes. 

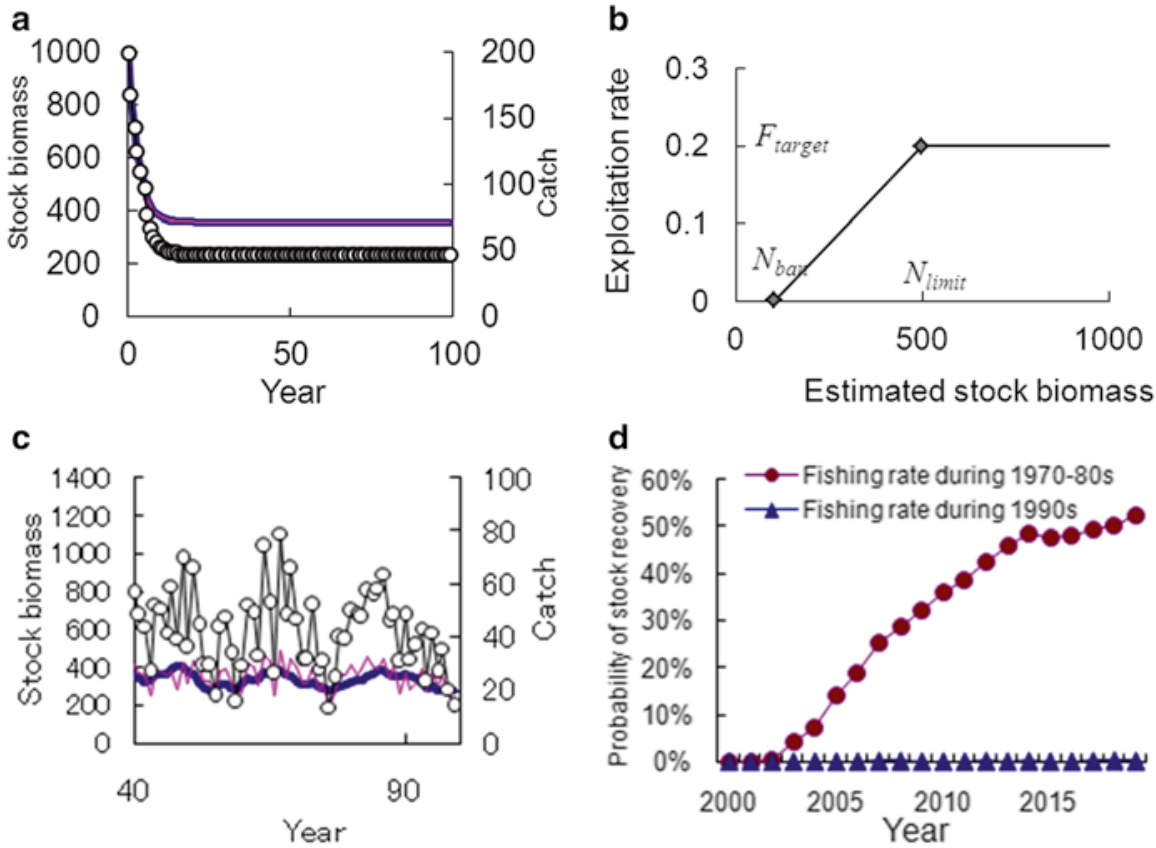

Fig. 9.3 Numerical examples of adaptive fisheries management using the same mathematical model as Fig. 9.1. (a) Trends of stock (bold line) and catch (circles) under constant recruitment ratio and constant exploitation rate (exploitation rate $F$ is 0.2 ). (b) The rule to determine the exploitation rate $(F)$ for the total allowable catch as a function of recent estimate of stock biomass. (c) Trends of stock (bold line) and catch (circles) under the rule given by panel b with estimation errors in stock abundance (estimated stock is shown by thin line) and process errors. (d) Probability of stock recovery under exploitation rate during 1970-1980s and exploitation rate during 1990s in a Japanese chub mackerel fishery (Kawai et al. 2002)

On the other hand, Fig. 9.3b illustrates the biological allowable catch decision rule, whereby the exploitation rate has been adjusted in accordance with estimated stock values in recent years to determine the Total Allowable Catch (TAC). Here the exploitation rate during a period of high stock is deliberately and mistakenly set at twice that in Fig. 9.3a. Because the true stock value is not known, the possibility of such a mistake cannot be ignored. Even in such a case, selective fishing can be avoided and relatively stable catches can be maintained as shown in Fig. 9.3c because the exploitation rate is lowered as the resources decrease.

Stock trends shown in Fig. 9.3a may be unrealistic because errors in the recruitment process are ignored. The figure shows a deterministic process whereby the stock trends are monotonically increasing or decreasing. Figure 9.3b, c seem more realistic, as these are stochastic processes incorporating process errors. Therefore, as shown in Fig. 9.3d, the vertical axis is the probability of stock recovery (to a particular level) instead of the stock biomass in Japanese stock assessment under various scenarios (Kawai et al. 2002). 
In Fig. 9.3, three types of uncertainty are considered. One is measurement error; an example is the disagreement between the thick and thin lines in Fig. 9.3c. This occurs because the true stock is unknown. The second is process error, and an example is the zigzag changes in stock in Fig. 9.3c, which are caused because the intrinsic rate of natural increase fluctuates every year. This is ignored in Fig. 9.3a. The third is operational error. This means that the actual exploitation rate varies slightly from the exploitation rate set in Fig. 9.3b. In risk management, it is necessary to consider any or all of these three types of uncertainty.

In addition, we should recognize the lack of full certainties relating to the mechanism of resource change. This is not considered in the management plan in Fig. 9.3. Uncertainties consist of those not predicted by the manager, those predicted but not clearly considered in the management plan, and those considered in risk management. The latter are uncertainties within the scope of the management plan's assumptions. However, not all uncertainties can be considered.

In Fig. 9.3c, numerical calculations are conducted by taking uncertainty into account and withdrawing random numbers. Every time a random number is withdrawn, therefore, a different result is obtained. In the case of the exploitation rate rules of Fig. 9.3b, of 100 numerical calculations, the minimum stock became lower than $300 \mathrm{t}$ twice, and the minimum catches became lower than $20 \mathrm{t}$ six times during 100 years, with no measures taken to prohibit fishing. An important role of risk management is to set some situations to be avoided, evaluate the probability (risks) of them occurring, and make a management plan to ensure that they will be held within allowable limits.

In conventional marine resources management, general predictions were made ignoring uncertainties such as those shown in Fig. 9.3a, and a management plan to obtain the most desirable situation was devised. Such a plan was unable to cope with uncertainty. The predictions made in such a management plan will be different from actual future results.

Since we used a stochastic model, we cannot describe unique future projections for each management scenario. Therefore, we calculate the risk of management failure from a large number of simulation results as shown in Fig. 9.3d. Note that the vertical axis of Fig. 9.3d is the risk of management failure, instead of the future stock abundance.

\subsection{Do Not Make a Single Prediction (Japanese Deer Protection Management Plan)}

Populations of Japanese deer have increased too much in numerous locations in Japan, posing a social problem. To address this issue, mathematical models of population dynamics were made. For example, the Forest Life Section of the Forestry and Forest Products Research Institute created a simple simulation program to manage deer, as shown in Fig. 9.4. Calculations were made with an Excel program, Simbambi, developed by Dr. Horino, section chief of the institute. The initial Simbambi was designed to make a general prediction for a specific capture policy as shown in 

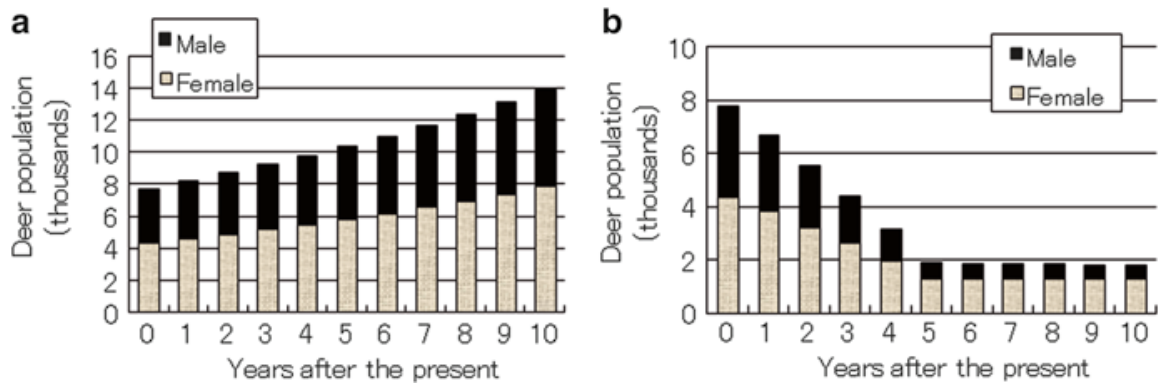

Fig. 9.4 Projection of deer population of Mt. Goyozan under two different scenarios: (a) No capture and (b) 800 individuals are caught every year until the 5th year and 190 individuals are caught every year from the 6 th year

Fig. 9.4, without considering the aforementioned uncertainty, and was a numerical calculation for the region of Mt. Goyozan, Iwate Prefecture. The simulated population size was subsequently changed from the actual size in order to create the present revised simulation program, Simbambi 4.1. The Japanese deer in Mt. Goyozan are considered excessive, and it is known that they must be captured or they would rapidly increase (Fig. 9.4a). In this model, the population size would increase limitlessly, but in reality it is believed that the food would be completely eaten, resulting in mass death. In fact, mass death is reported in such places as Cape Shiretoko, Nakajima Island in Lake Toya, and Kinkazan. Figure 9.4b shows predicted population size when 400 males and 400 females are captured every year for 5 years. In the region of Mt. Goyozan, the relationship between captured numbers and changes in population size is predicted when implementing the population management plan, the aim being to achieve coexistence between humans and deer.

Actually, however, the plan failed. From 1990, the number of deer captured was increased to 1,000 or more, and from 1993, the number of does captured was increased to about 1,000 more than planned for 5 years. The true population size is unknown, but it was probably underestimated, since it was possible to increase the numbers captured to that extent.

In the revised Simbambi 4.1 version of the population dynamics simulation program, the capture numbers are given by the user and the operational error is not considered, but the demographic probability fluctuation and environmental probability fluctuation are considered.

\subsection{Investigation for Only One Year Is Not Enough}

Survival and reproductive rates among wildlife are not the same every year, and during "strong year classes," once in several years, wild animals leave many offspring, but the numbers may also be greatly decreased due to "catastrophe" every few years. In the case of Japanese deer, the numbers increased too much resulting in mass death in such spatially limited habitats as the island of Nakajima in Lake Toya. 
During a year of extraordinarily heavy snowfall in the Meiji era, moreover, deer did not survive the winter, and mass death was recorded across the whole of Hokkaido. In the last 30 years of the twentieth century, however, no mass death occurred. One reason was the reduced frequency of heavy snowfall, but another reason may have been that expanded afforestation increased mixed forests of conifers and broadleaved trees, allowing deer to survive the winter even in a year of heavy snowfall.

To return to the topic of fish population management, another example worth noting with regard to annual fluctuation is that of char. These fish are listed as an endangered species because the population is divided into separate groups due to river repair works including dams. In the case of white-spotted char (Salvelinus leucomaenis), part of the population goes down to the sea and returns. However, if dam-like structures are constructed downstream, the char are prevented from going upstream (Morita and Yokota 2002). It is a problem if the population is maintained by the breeding of individuals remaining in the rivers. The recruitment rate fluctuates every year, and the survival rate to 1 year old fluctuates from 2 to $15 \%$. If the estimation of the survival rate is correct, the population size increases when the survival rate is $5 \%$ or higher, and it tends to decrease when the rate is lower than $5 \%$.

If it is assumed that the survival rate of the year investigated continues every year, future predictions may differ greatly from reality. If the recruitment rate (product of reproduction rate and initial survival rate to the age at recruitment, usually 1 year old) is underestimated, it will be assessed that the population of this species of char will become extinct. This will be the case whether or not new fisheries business is conducted in the location. If the recruitment rate is overestimated, it will be assessed that the survival of the population will not be affected even if some load is imposed by the business.

But both assessments are improper. As indicated earlier, reproductive rates and initial survival rates for wildlife often fluctuate every year. It is impossible to estimate reproductive rates and survival rates by investigating only one year, as is usually the case in environmental impact assessments. Even if a researcher conducts an investigation for five years, quantitative assessment may not be possible.

What should we do then? When assessing environmental impacts, as well as wildlife population levels, it is important to conduct not only a one-year investigation, but also to undertake subsequent post-investigation and reviewing, and other follow-up. Where population increases or decreases are concerned, various conservation measures should be prepared in advance, and the appropriate measures should then be taken in response to events as they evolve. This is the idea of adaptive management described above.

\subsection{Explanation of Formulae}

1. Relationship between stock biomass and its surplus production according to MSY theory-Fig. 9.1

The ordinary differential equation related to time $\mathrm{t}$ was made as follows:

$$
d N / d t=r(1-N / K) N-C
$$


where $N$ is stock, $C$ catches, and $r$ and $K$ are positive constants called intrinsic rate of natural increase and carrying capacity, respectively. We assumed $r=0.2$ and $K=1,000$.

2. Example of numeric calculation of adaptive marine resource management—Fig. 9.3

$$
N(t+1)=[N(t)-C(t)] \exp [r(t)-k\{N(t)-C(t)\}]
$$

where $k$ is a positive constant indicating the magnitude of density effect on the recruitment rate. $r(t)$ is not constant, but is given by:

$$
r(t)=\rho r(t-1)+(1-\rho) r *\left[1+\sigma_{\mathrm{r}} \varepsilon_{\mathrm{r}}(t)\right]
$$

where $\rho$ is the magnitude of autocorrelation in $r(t), \mathrm{r}^{*}$ is a positive constant implying the averager; $\sigma_{\mathrm{r}}$ and $\varepsilon_{\mathrm{r}}$ are the magnitude of error in the recruitment process and the random variable between -1 and +1 .

The exploitation rate $F(t)$ is determined by:

$$
F(t)=\left\{\begin{array}{c}
0 \text { if } \tilde{N}(t)<N_{\text {ban }} \\
F_{\text {target }} \frac{\tilde{N}(t)-N_{\text {ban }}}{N_{\text {limit }}-N_{\text {ban }}} \text { if } N_{\text {ban }}<\tilde{N}(t)<N_{\text {limit }} F \\
F_{\text {target }} \text { if } \tilde{N}(t)>N_{\text {limit }}
\end{array}\right.
$$

where $\tilde{N}(t)$ is the estimated stock biomass. We give it as:

$$
\tilde{N}(t)=N(t)\left[1+\sigma_{\mathrm{N}} \varepsilon_{\mathrm{N}}(t)\right]
$$

where $\sigma_{\mathrm{N}}$ and $\varepsilon_{\mathrm{N}}$ are the magnitude of estimation error and the random variable between -1 and +1 . Catch in year $t$, denoted by $C(t)$, is given by:

$$
C(t)=N(t) F(t)\left[1+\sigma_{\mathrm{F}} \varepsilon_{\mathrm{F}}(t)\right]
$$

where $\sigma_{\mathrm{F}}$ and $\varepsilon_{\mathrm{F}}$ are the magnitude of operational error in fishery and the random variable between -1 and +1 .

We set $r=0.2, K=N(0)=1,000 . \varepsilon_{\mathrm{N}}(\mathrm{t}), \varepsilon_{\mathrm{F}}(\mathrm{t})$ and $\varepsilon_{\mathrm{r}}(\mathrm{t})$ are uniform random numbers between -1 and +1 , and $\sigma_{\mathrm{N}}, \sigma_{\mathrm{F}}$, and $\sigma_{\mathrm{r}}$ were assumed to be 80,30 , and $10 \%$, respectively, in Fig. 9.3c, d. $\rho$ was assumed to be 0.7 in Fig. 9.3c, d. In the case of Fig. 9.3a, they were all made 0.

3. Concept of Japanese deer protection management-Fig. 9.4

The population size of the male and female deer of $i$ years old in year $t$ are denoted by $N_{i}{ }^{\circ}(\mathrm{t})$ and $N_{i}^{\gamma}(\mathrm{t})$, respectively, and $i=0,1,2,3$, and $4+;$ those aged 4 or older are handled together for simplicity (this is different from the Simbambi program). The population size of the following year is expressed by the following formulae.

$$
N_{0}{ }^{\dagger}(t)=N_{0}{ }^{\hat{\gamma}}(t)=\sum\left[N_{i}{ }^{\dagger}(t) m_{i}\right] / 2
$$


Table 9.1 Parameter values used for Fig. 9.4

\begin{tabular}{llllll}
\hline Age $i$ & 0 & 1 & 2 & 3 & 4 \\
Reproduction rate $m_{i}(\%)$ & 0 & 0 & 20 & 40 & 45 \\
Selective catch coefficient $f_{i}$ & 0.6 & 1 & 1 & 1 & 1 \\
Survival rate of males $p_{i}^{\circ}(\%)$ & 70 & 86 & 90 & 93 & 93 \\
Survival rate of females $p_{i}{ }^{\circ}(\%)$ & 70 & 82 & 88 & 90 & 90 \\
\hline
\end{tabular}

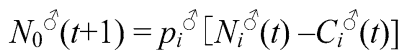

$$
\begin{aligned}
& N_{i}^{\circ}(t+1)=p_{i}{ }^{+}\left[N_{i}^{+}(t)-C_{i}^{+}(t)\right] \\
& N_{i}^{\widehat{\partial}}(t+1)=p_{i}^{\hat{\partial}}\left[N_{i}^{\hat{\partial}}(t)-C_{i}^{\hat{\partial}}(t)\right] \\
& N_{4+}{ }^{\circ}(t+1)=p_{4}{ }^{q}\left[N_{3}{ }^{\circ}(t)+N_{4+}{ }^{\circ}(t)-C_{3}{ }^{\circ}(t)-C_{3}{ }^{\circ}(t)\right] \\
& N_{4+}{ }^{\hat{\partial}}(t+1)=p_{4}{ }^{\hat{\lambda}}\left[N_{3}{ }^{\hat{\partial}}(t)+N_{4+}{ }^{\hat{\partial}}(t)-C_{3}{ }^{\hat{\partial}}(t)-C_{4+}{ }^{\hat{\partial}}(t)\right] \\
& C_{i}^{q}(t)=C^{q}(t) N_{i}^{q}(t) f_{i} / \quad \sum\left[N_{j}^{\uparrow}(t) f_{i}\right]
\end{aligned}
$$

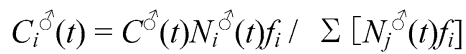

Where $m_{i}$ is pregnancy rate of $i$ year-old, $f_{i}$ is selective catch coefficient of $i$ year-old, $C^{\dagger}(t)$ and $C^{\delta}(\mathrm{t})$ are total numbers of males and females captured in year $t$, respectively. We assumed selective catching considering a high possibility of not capturing sub-adult animals. It was assumed that natural death would occur for individuals after they were captured and reduced in number (Table 9.1).

\subsection{Conclusion}

We need the concept of risk in fisheries and wildlife management because of several kinds of uncertainties. Traditional theory of maximum sustainable yield ignores such uncertainties and is not convincing for fishery operators, as biomass of fishery resources usually fluctuates remarkably. Risk analysis using stochastic models is useful and more convincing, even though the true mechanism of population dynamics is still unknown. We need risk evaluation to show a prediction range with some allowance.

I recommend adaptive management as a method of coping with such uncertainty. Adaptive management can change the exploitation rate of fish/wildlife populations. It can reduce future uncertainties compared to a policy with a constant exploitation rate. Therefore, adaptive management is indispensable in fisheries and wildlife management. We do not make a single prediction, but show the risk of management failure, which is rarely eliminated because of uncertainties. We compare the risk of management failure between two scenarios in order to show the advantages of a recommended management policy. 
Open Access This article is distributed under the terms of the Creative Commons Attribution Noncommercial License which permits any noncommercial use, distribution, and reproduction in any medium, provided the original author(s) and source are credited.

\section{References}

Clark CW (1990) Mathematical bioeconomics: the optimal management of renewable resources, 2nd edn. Wiley, New York

Holling CS (1966) The functional response of invertebrate predators to prey density. Mem Entomol Soc Can 48:1-86

Kawai H, Yatsu A, Watanabe C, Mitani T, Katsukawa T, Matsuda H (2002) Recovery policy for chub mackerel stock using recruitment-per-spawning. Fish Sci 68:961-969

Matsuda H, Abrams PA (2004) Effects of predator-prey interactions and adaptive change on sustainable yield. Can J Fish Aq Sci 61:175-184

Matsuda H, Abrams PA (2006) Maximal yields from multi-species fisheries systems: rules for systems with multiple trophic levels. Ecol Appl 16:225-237

Matsuda H, Abrams PA (2013) Is feedback control effective for ecosystem-based fisheries management? J Theor Biol 339:122-128

Mori M, Katsukawa T, Matsuda H (2001) Recovery plan for the exploited species: southern bluefin tuna. Pop Ecol 43:125-132

Morita K, Yokota A (2002) Population viability of stream-resident salmonids after habitat fragmentation: a case study with white-spotted char (Salvelinus leucomaenis) by an individual based model. Ecol Model 155:85-94

Walters CJ (1986) Adaptive management of renewable resources. McMillan, New York 